INPLASY

PROTOCOL

To cite: Zhou et al. Feasibility of Superb Microvascular Imaging for Detecting Neovascularization of Carotid Plaques: A Meta-Analysis. Inplasy protocol 202070050. doi:

10.37766/inplasy2020.7.0050

Received: 13 July 2020

Published: 13 July 2020

Corresponding author: Cong Wang

wc027214@163.com

Author Affiliation:

The First Affiliated Hospital of Dalian Medical University

Support: NA

Review Stage at time of this submission: Preliminary searches.

Conflicts of interest: None.

\section{Feasibility of Superb Microvascular Imaging for Detecting \\ Neovascularization of Carotid Plaques: A Meta-Analysis}

Zhou, Y1; Wang, C22.

Review question / Objective: Carotid artery atherosclerosis is a major risk factor for ischemic stroke. Intraplaque neovascularization (IPN) can promote the rapid progress of plaque, induce bleeding, and lead to plaque rupture. Superb microvascular imaging (SMI) is as a novel Doppler technique that depicts low velocity blood flow without the use of a contrast agent. This meta-analysis tested the hypothesis that superb microvascular imaging (SMI) can detect intraplaque neovascularization (IPN) in individuals with systemic vasculopathy of the carotid artery compared with CEUS.

Condition being studied: Studies suggested that SMI may or may not detect neovascularization of carotid plaque with accuracy comparable to CEUS.

INPLASY registration number: This protocol was registered with the International Platform of Registered Systematic Review and Meta-Analysis Protocols (INPLASY) on 13 July 2020 and was last updated on 13 July 2020 (registration number INPLASY202070050). 
hypothesis that superb microvascular imaging (SMI) can detect intraplaque neovascularization (IPN) in individuals with systemic vasculopathy of the carotid artery compared with CEUS.

Condition being studied: Studies suggested that SMI may or may not detect neovascularization of carotid plaque with accuracy comparable to CEUS.

\section{METHODS}

Search strategy: PubMed, Web of Science, Cochrane Library, CISCOM, and CBM databases were searched without language restrictions. The keywords and MeSH terms used included [carotid] and [plaques or plaque or fatty streak or fibroatheroma] and [contrast-enhanced ultrasound or contrast-enhanced ultrasonography or contrast ultrasonography or ultrasound contrast imaging or CEUS] and [vulnerability or stability or neovascularization] and [superb microvascular imaging]. We also performed a manual search to find other potential articles.

Participant or population: Neovascularization of Carotid Plaques.

Intervention: SMI.

Comparator: CEUS.

Study designs to be included: Clinical cohort study.

Eligibility criteria: (1) the study design was a clinical cohort study, (2) the study compared CEUS and SMI for detecting IPN, (3) intraplaque microvascular flow (IMVF) was graded, and (4) published data in the row $x$ column tables was sufficient for the odds ratio.

Information sources: PubMed, Web of Science, Cochrane Library, CISCOM, and CBM databases were searched without language restrictions.

Main outcome(s): Number of intraplaque microvascular flow (IMVF).
Quality assessment / Risk of bias analysis: The methodological quality of each study was independently assessed by two researchers according to a tool for the quality assessment of methodological index for non-randomized studies (MINORS).

Strategy of data synthesis: The STATA version 15.1 software (Stata Corporation, College Station, TX, USA) was used for meta-analysis. We calculated the pooled summary odds ratio (OR) and its 95\% confidence interval (Cl). The Cochran's Qstatistic and 12 test were used to evaluate potential heterogeneity between studies. If the Q-test showed a P50\%, indicating significant heterogeneity, and the random effect model was employed or if heterogeneity was not significant, the fixed-effects model was used. Sensitivity analysis was performed to evaluate the influence of a single study on the overall estimate. We used Begger's funnel plots and Egger's linear regression test to investigate publication bias.

Subgroup analysis: 1. Language; 2. Equipment.

Sensibility analysis: Sensitivity analysis was performed to evaluate the influence of a single study on the overall estimate.

Country(ies) involved: China.

Keywords: carotid plaque; contrastenhanced ultrasonography; intraplaque neovascularization; meta-analysis; superb microvascular imaging.

Contributions of each author:

Author 1 - Yang Zhou.

Author 2 - Cong Wang. 